\title{
Editorial: Blockade of PD-1 and PD-L1 restores defective innate immune responses in leukocytes from septic humans
}

\author{
By Peter A. Ward ${ }^{1}$ and Fatemeh Fattahi \\ Department of Pathology, University of Michigan Medical School, Ann Arbor, Michigan, USA \\ RECEIVED AUGUST 23, 2016; REVISED SEPTEMBER 16, 2016; ACCEPTED SEPTEMBER 19, 2016. DOI: 10.1189/jlb.3CE0816-360R \\ - SEE CORRESPONDING ARTICLE ON PAGE 1237
}

\begin{abstract}
Sepsis in humans has been an enigma, with $>100$ clinical trials failing to result in an effective drug approved by the U.S. Food and Drug Administration. It has long been known that blood leukocytes from patients with progressive sepsis (clinically designated as severe sepsis or septic shock) have defective innate immune and inflammatory responses. In the report by Patera et al. [1], blood leukocytes (neutrophils, monocytes, CD8 ${ }^{+}$ $T$ cells, and NK cells) from septic patients expressed increased amounts of the "checkpoint" proteins that consist of a family of surface inhibitory molecules [programmed cell death 1 (PD-1), programmed cell death ligand 1 (PD-L1), CTLA-4, $B-$ and T-lymphocyte attenuator, etc.] linked to PD. In the new report, the emphasis is on PD-1 and PD-L1 on surfaces of blood leukocytes. When these cells were examined in vitro, they had defective functional responses (phagocytosis and release of cytokines and IFN- $\gamma$ ) and reduced amounts of granzyme $B$ and CD107a expression. In vitro addition of blocking antibodies to PD-1 or PD-L1 (checkpoint molecules) to blood leukocytes from septic humans restored innate immune responses. These data suggest that PD-1 and PD-L1 suppress important functional responses of blood neutrophils, monocytes, and various
\end{abstract}

Abbreviations: PD-1 = programmed cell death 1, , PD-L1/2 = programmed cell death ligand 1/2 lymphoid cells. The in vitro use of blocking antibodies to these checkpoint molecules appeared to restore functional responses that were suppressed during sepsis. Modest restoration in vitro has recently been reported in similar earlier studies [2], and checkpoint protein expression has been linked to mortality in septic mice [3]. These findings have implications for a new strategy to treat humans with sepsis. J. Leukoc. Biol.

100: 1229-1231; 2017.

$\mathrm{H}$ uman sepsis, occurring after infections with bacteria, viruses, fungi, or protozoa and conditions of "sterile sepsis" (as after trauma with hemorrhagic shock or chemical injury of liver), results in a chain of events in which the inflammatory and immune systems are activated in an attempt to control the early events of sepsis and to restore the tissue to its preinjury state. If all goes well in the setting of sepsis, the inflammatory and immune responses will recede, and tissues will be restored to their normal state. Sepsis, by definition, implies that something has gone wrong with control mechanisms that regulate these responses and that tissues are being further injured by excessive inflammatory and immune responses to intrinsic and extrinsic factors [4]. Some of the reasons for this harmful progression of events may be age related (and perhaps also gender associated), as well as a result of the presence of comorbidities (e.g., hypertension, diabetes mellitus, obesity, etc.) that interfere with recovery [5]. Another realization over the past few years is that "recovery" from sepsis may be subsequently associated with cognition loss, reduced quality of life, and death rates that when controlled for comorbidity conditions, may result in a death rate double that for a reference cohort [6]. There is no question that survival rates from sepsis have been improved over the past 2 decades. This relates to improved support measures, such as aggressive fluid management; intensive, early attempts to identify the infectious agent; and improved ventilation that reduces barotrauma. Obviously, there is a desperate need for effective therapeutic strategies in the early treatment of sepsis. As will be suggested, the data in the report by Patera et al. [1] may provide us with an important alternative therapeutic intervention for treatment of humans with sepsis, using mAb that appear to be safe for use in humans.

\section{Checkpoint proteins (PD-1 and PD-L1) on surfaces of leukocytes}

It has been known for some time that 2 molecules (PD-1 and PD-L1), which occur naturally on a variety of cells, negatively regulate immune responses in the setting of malignant tumors (melanomas or lung

1. Correspondence: Dept. of Pathology, University of Michigan Medical School, 1301 Catherine Rd., Ann Arbor, MI 48109-5602, USA.

E-mail: pward@umich.edu 
cancers that bear certain mutations) or viral infections (HIV, EBV, hepatitis C virus, etc.). These checkpoint molecules are normally present in small amounts on various cell types but are up-regulated in a variety of circumstances. PD-1 is found mainly on surfaces of $T$ cells, whereas PD-L1 is found on macrophages and APCs $[7,8]$. Sometimes both molecules are present on the same cells. In the setting of severe sepsis or septic shock, these checkpoint proteins often demonstrate increased expression and may be responsible for the progressive immunosuppression of sepsis, as well as defective mobilization of phagocytic cells (neutrophils, monocytes, macrophages) and $\mathrm{T}$ cells, all of which seriously compromise innate immune defenses against infectious microbes (Fig. 1) [9]. When this occurs, functional responses of neutrophils and monocytes are reduced (phagocytosis, release of proinflammatory mediators, etc.). In the case of $\mathrm{CD}^{+} \mathrm{T}$ cells and NK cells, increased expression of PD-1 and/or PD-L1 has been associated with defective IFN- $\gamma$ production and reduced levels of granzyme B and CD107a, implying serious dysfunction in innate and adaptive immune responses to infectious agents [10]. Probably the most surprising observation in the Patera et al. [1] report was the finding that when blood neutrophils, monocytes, $\mathrm{CD}^{+} \mathrm{T}$ cells, or NK cells from septic human donors were exposed in vitro to neutralizing antibodies to PD-1 and/or PD-L1, the functional responses of the leukocytes reappeared, which represents a most surprising but welcome outcome. When protein or peptide ligands bind to receptors on the surfaces of phagocytes, the ligand-receptor complex is usually internalized and transported to the endoplasmic reticulum where the ligand is hydrolyzed by acidic proteases,

PD-1 and PD-L1 Impair Innate Immune Responses of Septic Phagocytes

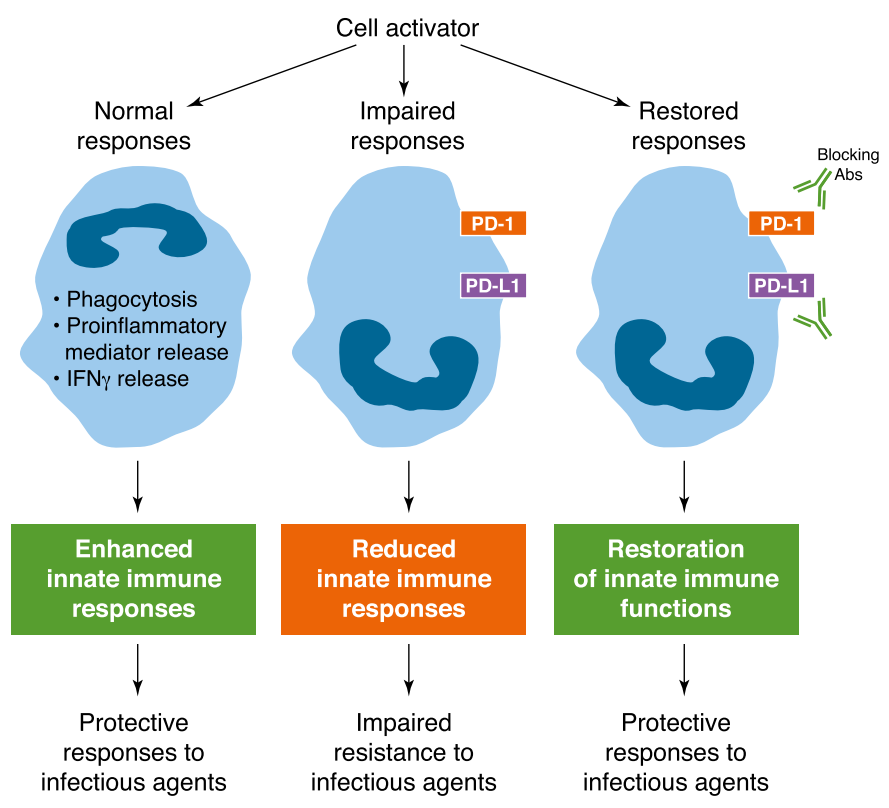

Figure 1. PD-1 and PD-L1 impair innate immune responses of septic phagocytes. (Left) The functional responses of phagocytes from mice or humans without sepsis. Activation of the cells with LPS or a variety or other agonists results in enhanced phagocytosis and release of proinflammatory mediators (cytokines and chemokines), as well as release of IFN- $\gamma$. These products promote inflammatory and innate and adoptive immune responses, resulting in protection against infectious agents. (Middle) Blood phagocytes obtained from septic humans or mice. In this situation, sepsis has led to up-regulation of PD-1 and PD-L1 on cell surfaces, resulting in suppressed innate immune and inflammatory responses, with consequent impairment of host defenses, especially related to infectious agents. (Right) Addition of neutralizing antibodies (Abs) to PD-1 or PD-L1 in vitro or in vivo reverses the suppressive state, causing restoration of innate immune and inflammatory responses of phagocytes in sepsis.

allowing some of the receptor to be recycled to the cell surface. Whether this is happening with checkpoint-antibody complexes remains to be determined.

\section{Clinical implications for checkpoint blockade in sepsis}

With the assumption that there are plans to expand the preliminary data substantially-suggesting that the blocking of antibodies to PD-1 and PD-L1 can reverse defective innate immune responses of blood leukocytes from septic humans-expanded and long-term studies will be critically important. Based on antibodies to PD-1 and PD-L1 in humans with advanced melanomas or with lung cancer [11], it seems that these antibodies are reasonably safe for use in humans, although this issue would have to be pursued with carefully designed phase I (safety) trials in septic patients. Furthermore, with the assumption that there will not be serious roadblocks in designing clinical trials and that there will be uncontestable evidence that the antibodies are safe and effective, this could dramatically change the strategy for treatment of humans with sepsis. In the past, there has been consideration for use of transfusion of various subtypes of leukocytes to reverse the immunosuppression of sepsis (reviewed in refs. $[12,13])$. However, given the numerous defects in phagocytes and in T cells in septic humans, such a strategy would be extremely labor intensive and would have the problem of whether transfused leukocytes could localize to critical areas of infection, as well as how long they would remain in the circulation. Furthermore, there remain questions of which type of infused cells would be most effective. Such concerns could be moot if early clinical trials indicate that antibodies to PD-1 and PD-L1 can be demonstrated to be clinically effective and safe in humans with sepsis. If such a situation were to occur, we would have a relatively simple, effective, and safe therapeutic approach to sepsis, for which evaluation could include daily clinical assessments and measurements by flow cytometry of blood neutrophils, monocytes, and $\mathrm{CD} 8^{+}$ $\mathrm{T}$ cells. If so, we might have a strategy that 
would reverse many decades of failed attempts to treat humans with sepsis effectively.

\section{Issues that should be addressed}

Obviously, the number of humans assessed was only 17, and the reference nonseptic group was 9. Expanded clinical studies will also subsequently be needed to address the following questions:

1. What are the mechanisms behind the ability of antibodies against PD-1 and/or PD-L1 to reverse in vitro the defective innate immune responses of blood leukocytes from septic mice and human patients?

2. What is the duration of the in vivo reversal in leukocyte defects in septic patients?

3. Is there homogeneity in terms of outcomes of septic patients treated with the antibodies to checkpoint inhibitors, or are the outcomes variable? If the latter is the case, what are the reasons?

4. Do companion studies in septic mice using the antibodies to PD-1 and PD-L1 reverse the ability of leukocytes to respond in vitro to various agonists (LPS, PMA, C5a, histones)?

5. Does use of the neutralizing antibodies to checkpoint molecules reverse the harmful effects of sepsis and/or improve survival in humans and/or interfere with development of long-term problems?

6. Does the intervention with antibodies to checkpoint proteins also attenuate the chain of events developing after sterile sepsis (hemorrhagic shock, etc.)?

7. Does intervention with antibodies in septic mice affect the development of immunosuppression?

\section{REFERENCES}

1. Patera, A. C., Drewry, A. M., Chang, K., Beiter, E. R., Osborne, D., Hotchkiss, R. S. (2016) Frontline science: defects in immune function in patients with sepsis are associated with PD-1 or PD-L1 expression and can be restored by antibodies targeting PD-1 or PD-L1. J. Leukoc. Biol. 100 XXX-XXX

2. Wang, J. F., Li, J. B., Zhao, Y. J., Yi, W. J., Bian, J. J., Wan, X. J., Zhu, K. M., Deng, X. M. (2015) Up-regulation of programmed cell death 1 ligand 1 on neutrophils may be involved in sepsis-induced immunosuppression: an animal study and a prospective case-control study. Anesthesiology 122, 852-863.

3. Huang, X., Chen, Y., Chung, C. S., Yuan, Z., Monaghan, S. F., Wang, F., Ayala, A. (2014) Identification of $\mathrm{B} 7-\mathrm{H1}$ as a novel mediator of the innate immune/proinflammatory response as well as a possible myeloid cell prognostic biomarker in sepsis. J. Immunol. 192, 1091-1099.

4. Rittirsch, D., Flierl, M. A., Ward, P. A. (2008) Harmful molecular mechanisms in sepsis. Nat. Rev. Immunol. 8, 776-787.

5. Martin, G. S., Mannino, D. M., Moss, M. (2006) The effect of age on the development and outcome of adult sepsis. Crit. Care Med. 34, 15-21.

6. Yende, S., Austin, S., Rhodes, A., Finfer, S., Opal, S., Thompson, T., Bozza, F. A., LaRosa, S. P., Ranieri, V. M., Angus, D. C. (2016) Long-term quality of life among survivors of severe sepsis: Analyses of two international trials. Crit. Care Med. 44, 1461-1467.

7. Loke, P., Allison, J. P. (2003) PD-L1 and PD-L2 are differentially regulated by Th and Th2 cells. Proc. Natl. Acad. Sci. USA 100, 5336-5341.

8. Dong, Z. Y., Wu, S. P., Liao, R. Q., Huang, S. M., Wu, Y. L. (2016) Potential biomarker for checkpoint blockade immunotherapy and treatment strategy. Tumour Biol. 37, 4251-4261.

9. Patil, N. K., Bohannon, J. K., Sherwood, E. R. (2016) Immunotherapy: a promising approach to reverse sepsis-induced immunosuppression. Pharmacol. Res. 111, 688-702.

10. Zelinskyy, G., Myers, L., Dietze, K. K., Gibbert, K., Roggendorf, M., Liu, J., Lu, M., Kraft, A. R., Teichgräber, V., Hasenkrug, K. J., Dittmer, U. (2011) Virus-specific CD8 $+\mathrm{T}$ cells upregulate programmed death-1 expression during acute friend retrovirus infection but are highly cytotoxic and control virus replication. J. Immunol. $\mathbf{1 8 7}$ 3730-3737.

11. Gandini, S., Massi, D., Mandalà, M. (2016) PD-L1 expression in cancer patients receiving anti PD-1/PD-L1 antibodies: a systematic review and meta-analysis. Crit. Rev Oncol. Hematol. 100, 88-98.

12. Delano, M. J., Ward, P. A. (2016) Sepsisinduced immune dysfunction: can immune therapies reduce mortality? J. Clin. Invest. 126, 23-31.

13. Minnich, D. J., Moldawer, L. L. (2004) Anticytokine and anti-inflammatory therapies for the treatment of severe sepsis: progress and pitfalls. Proc. Nutr. Soc. 63, 437-441.

\section{KEY WORDS:}

checkpoint proteins · sepsis · phagocytosis . immunosuppression 